\title{
LA PUBLICIDAD TELEVISIVA DIRIGIDA A MENORES EN ESPAÑA: SEGUIMIENTO DEL CÓDIGO PAOS
}

\section{TV Food Advertising to Minors in Spain: Monitoring of the PAOS Code}

\author{
Gdo. David RAMÍREZ PONCE \\ Universidad Rey Juan Carlos, España \\ E-mail: davidrami_95@hotmail.com \\ (iD) https://orcid.org/0000-0001-5223-2469
}

\author{
Dra. María Cruz LÓPEZ DE AYALA \\ Profesor titular. Universidad Rey Juan Carlos, España \\ E-mail: mariacruz.lopezdeayala@urjc.es \\ (iD) http://orcid.org/0000-0002-6989-866X
}

Fecha de recepción del artículo: 01/07/2019

Fecha de aceptación definitiva: 23/10/2019

\begin{abstract}
RESUMEN
En un contexto de preocupación por la fuerte prevalencia de la obesidad entre los niños españoles, el debate sobre la publicidad televisiva de alimentos para niños alcanza gran importancia. Aunque existen otros factores que influyen sobre la obesidad infantil, hay evidencias de que los mensajes publicitarios tienen un gran impacto sobre los hábitos alimenticios de los niños. Mediante un análisis de contenido, el objetivo de esta investigación es analizar y evaluar la eficacia del Código PAOS en 2018 como instrumento para mejorar la calidad publicitaria y promover hábitos de alimentación y modos de vida saludables. De este estudio se concluye: la elevada exposición de los niños menores de doce años a la publicidad de alimentos y bebidas, especialmente en los canales infantiles; una tendencia creciente en la presencia de publicidad insana en la franja de protección reforzada con respecto a 2012; que los anuncios de alimentos y bebidas apoyan su argumento de venta más en el incentivo que en el alimento; y, como conclusión general, que ha mejorado la calidad de este tipo de mensajes publicitarios a lo largo de los últimos seis años.
\end{abstract}

Palabras clave: Publicidad de alimentos y bebidas; menores; televisión; autorregulación; análisis de contenido.

\begin{abstract}
In a context of concern about the high prevalence of obesity among Spanish children, the debate on television advertising of food and drink products to children becomes more important. Although there are other factors that influence childhood obesity, there is evidence that commercial have a great impact on the eating habits of children. Through
\end{abstract}


a content analysis, the objective of this research is to analyze and assess the PAOS Code's effectiveness in 2018 as an instrument to improve commercial quality and promote eating habits and healthy lifestyles. This study concludes: the high exposure of children under the age of twelve to commercial of food and beverages, especially in children's channels; the presence of unhealthy commercial in the reinforced protection time slot is rising regarding 2012; food and drink commercials use selling argument supporting more in the incentive than in the food. Finally, the overall conclusion is that the quality of this type of commercial messages has improved over the last six years.

Key words: food and drinks advertising; minors; television; self-regulation; content analysis.

\section{Introducción}

La preocupación sobre la obesidad en los países desarrollados avanza a un ritmo frenético, a la par que el volumen de sus cifras entre adolescentes y niños. En España, aunque la evolución es decreciente, la obesidad y el sobrepeso afectan a un alto porcentaje de los menores: $23,2 \%$ de los niños entre seis y nueve años tienen problemas de sobrepeso y 18,1\% padecen obesidad (Estudio Aladino, 2015; y sitúa la prevalencia de este problema en España por encima de la media de los países de la OCDE (Aranceta et al., 2016).

Existen numerosas evidencias científicas que apuntan a una estrecha relación entre la exposición a la televisión y la obesidad (Dennison y Edmunds, 2008; Reilly et al., 2005; Viner y Cole, 2005), apuntándose a diferentes factores que explicarían esta asociación (Caroli et al., 2004; McGinnis, Goolman y Kraak, 2006; Ofcom, 2006; Livingstone, 2006; Cairns et al., 2012; Guthold et al., 2010; Royo-Bordonada y Rodríguez, 2015). No obstante, se enfatiza la influencia de la publicidad de alimentos procesados poco saludables como el más relevante (Wiecha et al., 2006), debido a que la mayor credulidad y menor experiencia de los menores (Pardo, Lázaro y García, 2012; Romero, 2016) les hace más susceptibles de verse influenciados por las estrategias persuasivas utilizadas en la comunicación comercial (Young, 1990).

Si añadimos que, como indican Royo-Bordonada y Rodríguez (2015), los niños no son capaces de distinguir la publicidad de la programación televisiva hasta los seis años de edad, no son conscientes de su finalidad comercial hasta los ocho años y no comienzan a ser conscientes de las técnicas persuasivas empleadas hasta los doce años, se hace imprescindible la colaboración de los medios de comunicación y autoridades responsables para controlar la calidad de los mensajes publicitarios de alimentos y bebidas dirigidos a menores. Con esta finalidad se puso en marcha en España el Código de Publicidad, Actividad, Obesidad y Salud, también conocido como Código PAOS. Sin embargo, León-Flández et al. (2017) pusieron de manifiesto las elevadas cifras de incumplimiento en 2012, cuestionando así su efectividad.

Seis años después de la recogida de estos datos, este trabajo propone su actualización y evalúa la eficacia de las normas del Código PAOS, analizando los contenidos de la publicidad de bebidas y alimentos dirigidos a los menores de edad en la franja de protección reforzada, con el objetivo de determinar la evolución de la calidad de las comunicaciones comerciales en los últimos años. 


\section{La protección de los menores en la publicidad en España: el código paos}

La preocupación de la Unión Europea por la protección de los menores de edad en el sector audiovisual se ha materializado en una serie de iniciativas, recomendaciones y políticas comunitarias que han actuado como acicate para que en España, al igual que en otros países de la Unión Europea, se hayan desarrollado normativas más concretas para defender y proteger los derechos de los más vulnerables. En el ámbito de la regulación de la publicidad de alimentos y bebidas dirigida a menores, cabe destacar la Ley General de Publicidad (LGP), la Ley General de la Comunicación Audiovisual (LGCA) (Ley $\mathrm{N}^{\circ} 7,2010$ ) o la Ley de seguridad alimentaria y nutrición (Ley $\left.\mathrm{N}^{\circ} 17,2011\right)$.

No obstante, la ambigüedad y vaguedad de algunos de los términos utilizados en la legislación española crea un vacío que deja su interpretación en manos de los anunciantes y los consumidores y crea ciertas dudas sobre su cumplimiento (Icmedia, 2014). Esto, junto al protagonismo creciente de los menores en las comunicaciones comerciales, ha dado lugar al desarrollo de una serie de códigos de conducta dirigidos a corregir la actividad publicitaria y a proteger al menor mediante sistemas de autorregulación publicitaria; opción que aparece recogida tanto en la LGCA como en la Ley de Seguridad Alimentaria y Nutrición. De este modo, se promueve que sean los propios anunciantes los que asuman su responsabilidad en este aspecto.

En España, desde 2005, está vigente el Código PAOS (Publicidad, Alimentación, Obesidad y Salud), que es el mecanismo de autorregulación de la publicidad alimentaria dirigida a los menores de doce años difundida a través de televisión y, desde 2013, a menores de quince en Internet. Actuando como un complemento del control legal, el código PAOS establece una serie de normas éticas que deberán cumplir las empresas adheridas al mismo.

El Código PAOS se reforzó en 2009 gracias al acuerdo de colaboración firmado por las principales cadenas de televisión, que se comprometieron a que la publicidad emitida en las franjas de protección al menor cumpliera con las normas deontológicas recogidas en él. Ese año se adhieren también al Código la Agencia Española de Consumo, Seguridad Alimentaria y Nutrición (a partir de ahora AECOSAN) y AUTOCONTROL, órgano que se encargará de controlar y supervisar que los mensajes publicitarios cumplen las normas establecidas por el Código.

\section{La efectividad del código paos}

El Código destaca que, desde su entrada en vigor en 2005 y hasta 2017, se han presentado treinta y una reclamaciones por incumplimiento de las normas éticas al Jurado de la Publicidad (Autocontrol, 2018). Este dato resulta contradictorio con respecto a lo que revelan otras investigaciones (León-Flández et al., 2017; Menéndez y Franco, 2009; Ponce-Blandón, Pabón-Carrasco y Lomas-Campos, 2017; Royo-Bordonada et al., 2016), en las que se pone en duda su eficacia.

Los estudios empíricos revelan que la publicidad insana ha seguido una tendencia creciente en los últimos años. González (2013) encontró que algo más de la mitad de los mensajes publicitarios de productos de alimentación con mayor número de inserciones publicitarias en 2006 eran poco saludables, dado su contenido en grasas, 
azúcares y sal, y 22,5\% se refieren a comida basura. En el mismo sentido, 60,9\% de 169 productos analizados en la investigación de Royo-Bordonada et al. (2016) pertenecían a productos no esenciales ni saludables, destacando las galletas, bizcochos, salsas y bebidas azucaradas.

Por su parte, Bermejo (2006) concreta que la presencia de nutrientes en los mensajes comerciales invierte la pirámide nutricional que recomienda la Sociedad Española de Nutrición Comunitaria (SENC). También Menéndez y Franco (2009) reafirman esta oferta de productos de escaso valor nutricional, sosteniendo que en su análisis fue casi nula la presencia de anuncios de frutas, verduras o pescados, alimentos necesarios en una dieta sana y equilibrada, así como de campañas que fomentan hábitos de vida saludables. Además, León-Flández et al. (2017) destacan el alto porcentaje de publicidad insana en los canales dirigidos al público infantil; mientras que Campos et al. (2016) señalan que la relación entre publicidad insana y sana mejoró entre 2007 y 2013 en estas cadenas temáticas; no obstante, debido a que también aumentó la presión publicitaria de estos productos entre ambas fechas, la situación no había mejorado.

Siguiendo con León-Flández et al. (2017), su estudio concluye que la efectividad de la autorregulación ha disminuido en el 2012 con respecto a cuatro años antes, especialmente en la franja de protección reforzada y en la norma 7 sobre el apoyo y promoción a través de personalidades populares entre el público infantil. Según su trabajo, el cumplimiento general del Código PAOS en el año 2012 fue de 11,7\%, con más del 95\% de los anuncios que incumplieron la norma relativa a la claridad en las presentaciones comparativas y casi el $80 \%$ que lo hicieron en relación con la información sobre los productos $(79,4 \%)$; por último, en torno a la cuarta parte vulneraron la norma relativa a promociones, sorteos y clubes infantiles y lo mismo con respecto a la promoción a través de personajes conocidos entre el público infantil.

Por otra parte, Menéndez y Franco (2009) destacan que más del 70\% de los anuncios de alimentos y bebidas hacen uso de la fantasía para explotar la imaginación del menor e incitar a la compra del producto. De la misma manera, es frecuente aquella publicidad que utiliza premios, regalos o sorteos para persuadir a los menores de que compren el producto. Y González (2013) encontró que esta estrategia es más frecuentemente utilizada entre los alimentos poco saludables.

Además, Ponce-Blandón et al. (2017) subrayan la casi nula presencia de información sobre los efectos negativos que implica el consumo excesivo de los alimentos anunciados debido a su bajo valor nutricional. El estudio también precisa que en los mensajes de estos anuncios se destaca el sabor de los productos y su asociación con la diversión, dejando en un segundo plano la información racional del producto como la referente a su calidad.

Si bien la inversión publicitaria en televisión en el año 2017 ha descendido respecto a 2012, la televisión sigue manteniendo una posición dominante y ocupa el puesto número seis del top diez de los sectores que más han invertido en publicidad en ese año (Infoadex, 2018). Paralelamente, aunque los niños españoles (4-12 años) son el colectivo de edad que menos televisión ve, después de adolescentes y jóvenes (13-24 años), estos siguen dedicando gran parte de su tiempo libre al consumo de este medio: dos horas y once minutos en 2018 (Barlovento Comunicación, 2019). Estos datos apuntan a la necesidad de analizar la evolución de la eficacia de las normas de Código PAOS y valorar si es necesaria una normativa que reclame una mayor respon- 
sabilidad por parte de los anunciantes del sector de bebidas y alimentos en televisión, con el fin de proteger a los menores de edad.

\section{Objetivos}

El objetivo general de este trabajo es evaluar el nivel de cumplimiento del Código PAOS a partir de una muestra de anuncios de alimentos y bebidas sin alcohol dirigidos a menores de doce años, dentro del horario de protección reforzada y emitidos en televisión en cuatro cadenas que emiten en abierto: dos generalistas, Antena 3 y Telecinco, y dos de índole infantil, Boing y Disney Channel.

Los objetivos específicos del estudio son:

1. Ponderar la presencia de alimentos no saludables frente a alimentos saludables en los anuncios.

2. Examinar los espacios temporales y cadenas en las que los menores de edad están más expuestos a este tipo de publicidad en España.

3. Identificar cuáles son las normas y recomendaciones del Código PAOS que más incumplimientos acumulan en los anuncios dirigidos al público infantil.

4. Determinar la evolución de la efectividad del Código en relación con los datos registrados en 2012, utilizando como referencia el trabajo de León-Flández et al. (2017).

\section{Metodología}

El trabajo utiliza la técnica de análisis de contenido aplicado a los anuncios de bebidas y alimentos dirigidos a menores de doce años, pertenezcan o no a empresas adheridas al Código PAOS, emitidos durante la franja horaria de protección al menor en cadenas de televisión que emiten en abierto.

Atendiendo a la cuota de pantalla alcanzada entre la población infantil en el mes de marzo (Barlovento Comunicación, 2018), se han seleccionado dos cadenas de carácter infantil y otras dos de cadenas generalistas para formar parte de la muestra: Boing (11,2\%) y Disney Channel $(9,7 \%)$, que ocuparon la segunda y la tercera posición entre las cadenas infantiles; y Telecinco (7,8\%) y Antena $3(7,5 \%)$, líderes entre las cadenas generalistas. Se ha desechado del análisis la cadena pública Clan, líder de audiencia entre niños de cuatro y doce años, debido a que no emite publicidad.

El período de análisis ha comprendido una semana que abarca desde el lunes, 9 de abril de 2018, al domingo 15, ambos incluidos. Este periodo temporal fue acotado una vez se identificó una elevada reiteración del conjunto de spots ya evaluado en el periodo delimitado. Esta semana no coincide con ningún periodo vacacional ni festivo, de manera que no se ve alterada ni la programación televisiva ni la franja horaria de protección reforzada.

Para seleccionar las unidades muestrales del estudio, es decir, los anuncios que tienen como destinatarios a los menores de doce años, se han seguido los tres criterios que se recogen en el Código (AECOSAN, 2013: 5):

- El tipo de producto alimenticio anunciado está objetivamente destinado al público infantil. 
- El mensaje publicitario está diseñado para atraer la atención de los menores de doce años.

- El producto publicitario está insertado en la franja horaria de protección reforzada y/o emitido en un soporte dirigido de forma mayoritaria al público infantil. Es preciso recordar que, en los días laborables, la protección reforzada es de 08:00 a 09:00 y de 17:00 a 20:00 horas, abarcando de 09:00 a 12:00 horas en los días festivos, sábados y domingos (Ley 7/2010, 31 de marzo, General de la Comunicación Audiovisual, artículo 7.2)

La muestra final está constituida por 530 anuncios dirigidos al público infantil, que se han extraído de un total de 743 anuncios de alimentos y bebidas sin alcohol registrados en las veintiséis horas de grabación de los cuatro canales analizados.

Variables del análisis

De las catorce normas éticas que recoge el código, en el análisis de contenido no se han tenido en cuenta la norma 1 y 2 (principios de legalidad y de lealtad), presuponiendo que la publicidad emitida cumple con la legislación vigente y con los criterios de buena fe. Tampoco se han estudiado las normas 12, 13 y 14 porque están relacionadas con la publicidad de alimentos y bebidas destinada a menores de 15 años en Internet. Por tanto, se ha analizado desde la norma 3 a la norma 11, teniendo en cuenta un total de 23 de las 32 recomendaciones.

1. Norma 3 de educación e información nutricional, que determina que la publicidad no debe fomentar hábitos de alimentación poco saludables (recomendación 1 , 2 y 3).

2. Norma 4 sobre la presentación de los productos, que no debe inducir a error sobre las características y beneficios del producto anunciado (recomendación 4, 5 y 6), ni aprovecharse de la imaginación del menor de edad, haciendo uso de la fantasía (recomendación 7), y evitar mensajes violentos y agresivos (recomendación 8).

3. Norma 5 de información sobre los productos, que tiene que utilizar un lenguaje acorde a la edad del público (recomendación 9).

4. Norma 6 sobre presión de ventas, referente a que la publicidad no debe persuadir a los menores a adquirir el producto o reclamarlo a otros, utilizando para ello obsequios, asegurando el éxito social o dando a entender que se adquieren las habilidades del personaje por la compra del producto; además deben aparecer claramente los precios, sin incluir conceptos como «solo» o «nada más» (recomendaciones 10, 11, 12 y 13).

5. Norma 7 de apoyo y promoción a través de personajes y programas, que no deben influir en su decisión de compra y limita su aparición en casos en los que se relacionen claramente con el producto o fomenten una alimentación saludable (recomendación 14, 14.1, 14.2 y 14.3). Tampoco aparecerán tele promociones de estos productos en programas dirigidos a menores de doce años, salvo para fomentar hábitos saludables y a través de la figura del presentador o personaje del programa (recomendación 15).

6. Norma 8 de identificación de la publicidad, que obliga a separar los contenidos comerciales de los contenidos informativos de los programas (recomendación 16).

7. Norma 9 acerca de presentaciones comparativas, que debe ser objetiva y clara para el menor de edad.

8. Norma 10 de promociones, sorteos, concursos y clubes infantiles, separando el incentivo del producto, utilizando un lenguaje acorde a la edad del público infantil, no 
inducir a error en el premio que debe ser acorde al público infantil. En tanto que los clubs infantiles deben seguir los criterios de interactividad, continuidad y exclusividad en la publicidad de estos productos (recomendación 10 y 21).

9. Norma 11 sobre seguridad, que no anime a los menores a usar de forma inadecuada o peligrosa el producto o a entrar en lugares extraños o hablar con desconocidos (menores de 15 años) (recomendación 22 y 23).

Para asegurar la comparabilidad con los datos del año 2012, se han utilizado como referencia las variables más relevantes empleadas por León-Flández et al. (2017) en su estudio:

1. Tipo de producto: bebidas o alimentos comestibles.

2. Contenido nutricional. Apoyándose en Kelly et al. (2010) y Whalen et al. (2018), los autores agruparon los alimentos en tres categorías según su contenido nutricional.

- Alimentos esenciales y saludables. Se incluye todos los productos ricos en nutrientes y bajos en calorías, como frutas y productos de fruta sin azúcares añadidos; panes, arroz, pasta y fideos; cereales y galletas para el desayuno con bajo contenido de azúcar y alto contenido de fibra; verduras y productos vegetales; leche baja en grasa / reducida en grasa, yogur, natillas y queso; carne y productos similares; pescado, legumbres, huevos y nueces y; sopas, ensaladas mixtas; alimentos para bebés; y agua embotellada.

- Alimentos no esenciales y no saludables. Aquellos productos altos en grasas, azúcares, y sal, como pasteles, magdalenas, galletas dulces, galletas sabrosas con alto contenido de grasa, tartas y pasteles y bollos; cereales con alto contenido de azúcar y / o bajo contenido de fibra; snacks, incluyendo patatas fritas, palomitas de maíz, barritas de comida, productos azucarados y nueces recubiertas de azúcar o sal; alternativas de carne y carne desmenuzada o maltratada y comidas congeladas; bebida de frutas con alto contenido en azúcar; leche entera, yogur, natillas, postres lácteos y queso con alto contenido de sal y grasa; helado y postres con altos contenidos en azúcares; chocolate y productos de confitería. Chuches y chicles; restaurantes/alimentos de comida rápida; mantequilla, mermelada, aceites, salsas con alto contenido de grasa; bebidas azucaradas; y alcohol.

- Categoría de alimentos misceláneos. Incluyen suplementos de vitaminas y minerales; té y café; servicios de entrega de comida a domicilio; supermercados que anuncian principalmente alimentos no esenciales; supermercados que anuncian principalmente alimentos esenciales; supermercados que no especifican la categoría de alimentos en la publicidad; y leches de fórmula para bebés y niños pequeños.

3. Características de la emisión:

- Día de la semana.

- Canal de televisión.

- La franja de emisión del anuncio, diferenciando tres periodos dentro de la franja de protección reforzada:

- Primera hora de la mañana (08:00 a 09:00 horas). 
- $\quad$ Tarde (17:00 a 20:00 horas).

- Fines de semana (09:00 a 12:00 horas).

4. El cumplimiento individual de las normas del Código de autorregulación se ha clasificado en tres posibles categorías:

- Cumplimiento, cuando respeta todas las recomendaciones recogidas en esa norma.

- No cumplimiento, cuando infringe una o más recomendaciones que aparecen en esa norma.

- Cumplimiento dudoso, en el caso de que exista dificultad para evaluar objetivamente el cumplimiento de alguna de las recomendaciones de la norma.

5. El cumplimiento general del Código PAOS. Se ha considerado que un anuncio es cumplidor cuando respeta todas las recomendaciones de las nueve normas analizadas, no cumplidor cuando se infringe una o más normas deontológicas y de cumplimiento dudoso en el caso que exista dificultad para evaluarlo objetivamente.

Tratamiento de los datos

Los contenidos que forman el marco muestral fueron grabados y almacenados en un disco duro. Posteriormente, estos se codificaron manualmente y fueron registrados en una base de datos con todas las variables del estudio codificadas, para su posterior análisis. Para probar la fiabilidad de los datos, los dos investigadores que codificaron los contenidos probaron el esquema con una muestra de diez anuncios. En esta fase no se identificaron discrepancias en el marco de codificación, por lo que se dio por definitivo.

\section{Resultados}

En primer lugar, se observa una diferencia significativa en el número de anuncios de alimentos y bebidas dirigidos a menores de doce años emitidos en las cadenas generalistas $(13,8 \%)$ y en las cadenas infantiles $(86,2 \%)$ (Véase tabla 1). De lunes a viernes, el público de estas cadenas ha estado expuesto a un total de 410 estos mensajes comerciales, lo que supone una media de 82 anuncios diarios; mientras que se han registrado 120 anuncios durante todo el fin de semana. El viernes acumula una emisión más elevada de este tipo de mensajes comerciales, con 103 anuncios.

Más de la mitad de los anuncios (56,6\%) se han emitido por la tarde, de 17:00 a 20:00. Y aunque la franja de protección reforzada de 08:00 a 09:00 ha sido la que menos mensajes comerciales registra (110), si ponderamos por número de horas, la media es ligeramente superior al resto. 
DAVID RAMÍREZ PONCE Y MARÍA CRUZ LÓPEZ DE AYALA

LA PUBLICIDAD TELEVISIVA DIRIGIDA A MENORES EN ESPAÑA: SEGUIMIENTO DEL CÓDIGO PAOS

Tabla 1. Características de los anuncios de alimentos y bebidas dirigidos a menores de doce años

\begin{tabular}{|c|c|c|c|c|c|c|c|c|c|c|}
\hline & \multicolumn{2}{|c|}{ BOING } & \multicolumn{2}{|c|}{$\begin{array}{c}\text { DISNEY } \\
\text { CHANNEL }\end{array}$} & \multicolumn{2}{|c|}{ ANTENA 3} & \multicolumn{2}{|c|}{ TELECINCO } & \multicolumn{2}{|c|}{ TOTAL } \\
\hline & $\mathrm{N}$ & $\%$ & $\mathrm{~N}$ & $\%$ & $\mathrm{~N}$ & $\%$ & $\mathrm{~N}$ & $\%$ & $\mathrm{~N}$ & $\%$ \\
\hline \multicolumn{11}{|c|}{ Día de la semana } \\
\hline Diario & 194 & 47,3 & 153 & 37,3 & 38 & 9,3 & 25 & 6,1 & 410 & 77,4 \\
\hline Lunes & 39 & 52,0 & 30 & 40,0 & 4 & 5,3 & 2 & 2,7 & 75 & 14,2 \\
\hline Martes & 36 & 51,4 & 23 & 32,9 & 7 & 10 & 4 & 5,7 & 70 & 13,2 \\
\hline Miércoles & 34 & 45,3 & 27 & 36,0 & 9 & 12 & 5 & 6,7 & 75 & 14,2 \\
\hline Jueves & 42 & 48,3 & 32 & 36,8 & 8 & 9,2 & 5 & 5,7 & 87 & 16,4 \\
\hline Viernes & 43 & 41,8 & 41 & 39,8 & 10 & 9,7 & 9 & 8,7 & 103 & 19,4 \\
\hline $\begin{array}{l}\text { Fin de } \\
\text { semana }\end{array}$ & 45 & 37,5 & 65 & 54,2 & 4 & 3,3 & 6 & 5 & 120 & 22,7 \\
\hline Sábado & 23 & 41,1 & 30 & 53,6 & 1 & 1,8 & 2 & 3,6 & 56 & 10,6 \\
\hline Domingo & 22 & 34,4 & 35 & 54,7 & 3 & 4,7 & 4 & 6,2 & 64 & 12,1 \\
\hline \multicolumn{11}{|c|}{ Tipo de alimento } \\
\hline Comida & 122 & 43,9 & 111 & 39,9 & 29 & 10,4 & 16 & 5,8 & 278 & 52,5 \\
\hline Bebida & 117 & 46,4 & 107 & 42,5 & 13 & 5,2 & 15 & 5,9 & 252 & 47,5 \\
\hline \multicolumn{11}{|c|}{ Contenido nutricional } \\
\hline Esencial & 58 & 40,3 & 68 & 47,2 & 11 & 7,6 & 7 & 4,9 & 144 & 27,2 \\
\hline No esencial & 181 & 46,9 & 150 & 38,9 & 31 & 8,0 & 24 & 6,2 & 386 & 72,8 \\
\hline Miscelánea & --- & ---- & ---- & ---- & ---- & ---- & ---- & ---- & ---- & ---- \\
\hline \multicolumn{11}{|c|}{ Franja de protección reforzada } \\
\hline 08:00 a 09:00 & 63 & 57,3 & 40 & 36,4 & 2 & 1,8 & 5 & 4,5 & 110 & 20,7 \\
\hline $17: 00$ a $20: 00$ & 131 & 43,7 & 113 & 37,7 & 36 & 12,0 & 20 & 6,6 & 300 & 56,6 \\
\hline 09:00 a 12:00 & 45 & 37,5 & 65 & 54,2 & 4 & 3,3 & 6 & 5 & 120 & 22,7 \\
\hline \multicolumn{11}{|c|}{ Total, anuncios de alimentos y bebidas destinados a: } \\
\hline $\begin{array}{l}\text { Conjunto } \\
\text { población }\end{array}$ & 239 & 32,2 & 218 & 29,3 & 155 & 20,9 & 131 & 17,6 & 743 & 100,0 \\
\hline $\begin{array}{l}\text { Menores de } \\
12 \text { años }\end{array}$ & 239 & 45,0 & 218 & 41,1 & 42 & 8,0 & 31 & 5,9 & 530 & 100,0 \\
\hline
\end{tabular}

Fuente: Elaboración propia

De todos los anuncios analizados, $72,8 \%$ se han clasificado dentro de la categoría de alimentos no esenciales y poco saludables, la mayoría en cadenas infantiles, de los cuales, los batidos (26,6\%) junto con la bollería industrial $(15,1 \%)$ han sido los productos más publicitados.

También se han encontrado notables diferencias en el contenido nutricional de los mensajes comerciales emitidos en las cuatro cadenas (véase tabla 2). La cadena 
que acumula un porcentaje más importante de anuncios de alimentos no esenciales y poco saludables sobre el total de los emitidos por la misma es la cadena generalista Telecinco: con 24 de los 31 anuncios publicitados correspondiendo a esta categoría (77,4\%). A continuación, se sitúa la cadena infantil Boing (75,7\%), Telecinco $(73,8 \%)$ y, por último, Disney Channel (68,8\%). En conjunto para las cadenas infantiles, la relación entre alimentos no esenciales y no saludables y a los alimentos esenciales y saludables es de $72,4 \%$ frente al $27,5 \%$. Mientras que, en las cadenas generalistas, esta relación es de $75,3 \%$ frente al $24,7 \%$.

Tabla 2. Contenido nutricional de los anuncios de alimentos y bebidas por cadenas.

Porcentajes horizontales

\begin{tabular}{|l|c|c|c|c|c|c|c|c|c|c|}
\hline & \multicolumn{2}{|c|}{ BOING } & \multicolumn{2}{c|}{$\begin{array}{c}\text { DISNEY } \\
\text { CHANNEL }\end{array}$} & \multicolumn{2}{c|}{ ANTENA3 } & \multicolumn{2}{c|}{ TELECINCO } & \multicolumn{2}{c|}{ TOTAL } \\
\cline { 2 - 12 } & $\mathrm{N}$ & $\%$ & $\mathrm{~N}$ & $\%$ & $\mathrm{~N}$ & $\%$ & $\mathrm{~N}$ & $\%$ & $\mathrm{~N}$ & $\%$ \\
\hline $\begin{array}{l}\text { Alimentos } \\
\text { esenciales y } \\
\text { saludables }\end{array}$ & 58 & 40,3 & 68 & 47,2 & 11 & 7,6 & 7 & 4,9 & 144 & 27,2 \\
\hline Leche & 37 & 47,4 & 37 & 47,4 & 2 & 2,6 & 2 & 2,6 & 78 & 14,7 \\
\hline $\begin{array}{l}\text { Alimentos para } \\
\text { bebés: leches } \\
\text { infantiles }\end{array}$ & 21 & 37,5 & 31 & 55,4 & 4 & 7,1 & ---- & ---- & 56 & 10,6 \\
\hline $\begin{array}{l}\text { Carne: carne } \\
\text { de cerdo baja } \\
\text { en grasa }\end{array}$ & ---- & ---- & ---- & ---- & ---- & ---- & 5 & 100,0 & 5 & 0,9 \\
\hline Pasta & ---- & ---- & ---- & ---- & 2 & 100,0 & ---- & ---- & 2 & 0,4 \\
\hline Fruta & ---- & ---- & ---- & ---- & 3 & 100,0 & ---- & ---- & 3 & 0,6 \\
\hline $\begin{array}{l}\text { Alim. no } \\
\text { esenciales y } \\
\text { no saludables }\end{array}$ & 181 & 46,9 & 150 & 38,9 & 31 & 8,0 & 24 & 6,2 & 386 & 72,8 \\
\hline $\begin{array}{l}\text { Bollería } \\
\text { industrial }\end{array}$ & 43 & 53,8 & 37 & 46,2 & ---- & ---- & ---- & ---- & 80 & 15,1 \\
\hline $\begin{array}{l}\text { Queso con } \\
\text { alto contenido } \\
\text { de sal y grasa }\end{array}$ & 36 & 57,1 & 25 & 39,7 & 1 & 1,6 & 1 & 1,6 & 63 & 11,9 \\
\hline $\begin{array}{l}\text { Batidos de } \\
\text { chocolate }\end{array}$ & 66 & 46,8 & 52 & 36,9 & 10 & 7,1 & 13 & 9,2 & 141 & 26,6 \\
\hline $\begin{array}{l}\text { Galletas con } \\
\text { alto contenido } \\
\text { de grasa }\end{array}$ & 8 & 44,4 & 7 & 38,9 & 3 & 16,7 & ---- & ---- & 18 & 3,4 \\
\hline Chocolate & 12 & 30,0 & 14 & 35,0 & 7 & 17,5 & 7 & 17,5 & 40 & 7,5 \\
\hline \begin{tabular}{l} 
Snacks \\
\hline Golosinas
\end{tabular} & 16 & 48,5 & 15 & 45,4 & 2 & 6,1 & ---- & ---- & 33 & 6,2 \\
\hline $\begin{array}{l}\text { Alimentos } \\
\text { misceláneos }\end{array}$ & ---- & ---- & ---- & ---- & ---- & ---- & --- & --- & ---- & ---- \\
\hline Totales & 239 & 45,0 & 218 & 41,1 & 42 & 8,0 & 31 & 5,9 & 530 & 100,0 \\
\hline
\end{tabular}

Fuente: Elaboración propia 
Centrándonos en el cumplimiento individual de las normas y recomendaciones del Código PAOS (véase tabla 3), la norma 6 (presión de ventas) es la que ha presentado el porcentaje más alto de vulneraciones (81,1\%). Esta violación de la norma consiste principalmente en el llamamiento directo al público infantil a través de la aparición de otros menores y en dar más importancia al incentivo que a la información nutricional del producto anunciado (recomendaciones 10 y 11).

La segunda norma más incumplida $(43,4 \%)$ es la 4 , referida a la presentación de los productos anunciados. De estos, 18,5\% han hecho uso de la fantasía, explotando la imaginación de los menores a través de efectos visuales, sonoros y escritos; $18,3 \%$ han promocionado el producto, asociando el sabor como una característica única; $17 \%$ han inducido a error al destinatario sobre las características nutricionales del producto mediante las presentaciones visuales, escritas y sonoras; y 12,6\% han inducido a error al destinatario acerca de los beneficios del producto publicitado.

En cuanto a la norma 7, de 130 anuncios que han utilizado personajes, reales o ficticios para promocionar los productos anunciados, únicamente quince la han incumplido. Se han considerado diez anuncios de cumplimiento dudoso porque, como en el caso de Puleva, existe una relación directa de la mascota con el producto promocionado; y, aunque 35\% de los niños de 11 y 12 años tienen un perfil en redes sociales (Garmendia et al., 2018), los influencers que aparecen en el anuncio de Nocilla no necesariamente son conocidos por estos.

Por el contrario, 93,4\% de los anuncios analizados han incluido mensajes que fomentan hábitos saludables, cumpliendo la norma 3 sobre la educación e información nutricional. La norma 11, referida a la seguridad, y la norma 5, respecto a la información sobre los productos publicitados, se han cumplido en todos los anuncios analizados, no mostrando situaciones peligrosas o inadecuadas y utilizando un lenguaje claro y sencillo adecuado para el público infantil. Además, todos los anuncios que han ocupado la primera y la última posición dentro de cada bloque publicitario se han ajustado a la norma 8 , porque han separado claramente los contenidos comerciales de la programación.

No se ha identificado presentaciones publicitarias comparativas en el análisis que puedan dar lugar a incumplimientos de la norma 9 y tampoco se han incluido sorteos, promociones o concursos o se ha hecho referencia a clubes infantiles (norma 10).

El 81,8\% de anuncios han vulnerado este Código en alguno de sus puntos, 16\% ha cumplido todas las normas analizadas y $2.9 \%$ se ha considerado de cumplimiento dudoso debido a la dificultad de valorar objetivamente alguna de las normas. El incumplimiento del Código ha sido mayor en las cadenas infantiles: $91,2 \%$ frente a $8,8 \%$ de las cadenas generalistas.

El aspecto más significativo del cumplimiento global del Código ha estado vinculado al contenido nutricional de los anuncios de alimentos y bebidas analizados. De 85 anuncios que han cumplido el código (16\%), 83 anuncios pertenecían a la categoría de alimentos saludables y esenciales; mientras que solo 61 (14.2\%) de 430 anuncios que han incumplido el código se han clasificado dentro de esa categoría, perteneciendo la gran mayoría $(85,8 \%)$ a la clase de alimentos no esenciales y no saludables. 
DAVID RAMÍREZ PONCE Y MARÍA CRUZ LÓPEZ DE AYALA

LA PUBLICIDAD TELEVISIVA DIRIGIDA A MENORES EN ESPAÑA:

SEGUIMIENTO DEL CÓDIGO PAOS

Tabla 3. Cumplimiento de las normas y recomendaciones del Código PAOS por los anuncios de alimentos y bebidas dirigidos a menores de doce años

\begin{tabular}{|c|c|c|c|c|c|c|}
\hline & \multicolumn{2}{|c|}{ Cumplimiento } & \multicolumn{2}{|c|}{$\begin{array}{c}\text { No } \\
\text { Cumplimiento }\end{array}$} & \multicolumn{2}{|c|}{$\begin{array}{l}\text { Cumplimiento } \\
\text { dudoso }\end{array}$} \\
\hline & $\mathrm{N}$ & $\%$ & $\mathrm{~N}$ & $\%$ & $\mathrm{~N}$ & $\%$ \\
\hline Norma 3: educación e información nutricional & 495 & 93,4 & ---- & ---- & 35 & 6,6 \\
\hline $1^{\mathrm{a}}$ recomendación & 495 & 93,4 & ---- & ---- & 35 & 6,6 \\
\hline $2^{\mathrm{a}}$ recomendación & 495 & 93,4 & ---- & ---- & ---- & ---- \\
\hline $3^{\text {a }}$ recomendación & 530 & 100,0 & ---- & ---- & ---- & ---- \\
\hline Norma 4: presentación de los productos & 176 & 33,2 & 230 & 43,4 & 124 & 23,4 \\
\hline $4^{\text {a }}$ recomendación & 176 & 100,0 & 90 & 17,0 & 63 & 50,8 \\
\hline $5^{\text {a }}$ recomendación & 176 & 100,0 & 67 & 12,6 & 34 & 27,4 \\
\hline $6^{\text {a }}$ recomendación & 176 & 100,0 & 97 & 18,3 & 11 & 8,9 \\
\hline $7^{\mathrm{a}}$ recomendación & 176 & 100,0 & 98 & 18,5 & 36 & 29,0 \\
\hline $8^{\text {a }}$ recomendación & 530 & 100,0 & ---- & ---- & ---- & ---- \\
\hline Norma 5: información sobre los productos & 530 & 100,0 & ---- & ---- & ---- & ---- \\
\hline $9^{\text {a }}$ recomendación & 530 & 100,0 & ---- & ---- & ---- & ---- \\
\hline Norma 6: presión de ventas & 100 & 18,9 & 430 & 81,1 & ---- & ---- \\
\hline $10^{\mathrm{a}}$ recomendación & 100 & 100,0 & 313 & 72,8 & ---- & ---- \\
\hline $11^{\mathrm{a}}$ recomendación & 100 & 100,0 & 164 & 38,1 & ---- & ---- \\
\hline $12^{\mathrm{a}}$ recomendación & 100 & 100,0 & 42 & 9,8 & ---- & ---- \\
\hline $13^{\mathrm{a}}$ recomendación & ---- & ---- & ---- & ---- & ---- & ---- \\
\hline Norma 7: personajes y programas & 105 & 19,8 & 15 & 2,8 & 10 & 1,9 \\
\hline $14.1^{\mathrm{a}}$ recomendación & 105 & 100,0 & 15 & 100,0 & ---- & ---- \\
\hline $14.2^{\mathrm{a}}$ recomendación & ---- & ---- & ---- & ---- & 10 & 100,0 \\
\hline $14.3^{\mathrm{a}}$ recomendación & ---- & ---- & ---- & ---- & ---- & ---- \\
\hline $15^{\mathrm{a}}$ recomendación & ---- & ---- & ---- & ---- & ---- & ---- \\
\hline Norma 8: Identificación de la publicidad & 59 & 11,1 & ---- & ---- & ---- & ---- \\
\hline $16^{\mathrm{a}}$ recomendación & 59 & 100,0 & ---- & ---- & ---- & ---- \\
\hline Norma 9: presentaciones comparativas. & ---- & ---- & ---- & ---- & ---- & --- \\
\hline $\begin{array}{l}\text { Norma 10: promociones, sorteos, concursos y } \\
\text { clubes infantiles. }\end{array}$ & ---- & ---- & ---- & ---- & ---- & ---- \\
\hline Norma 11: seguridad. & 530 & 100,0 & ---- & ---- & ---- & ---- \\
\hline $22^{\mathrm{a}}$ recomendación & 530 & 100,0 & ---- & ---- & ---- & ---- \\
\hline $23^{\mathrm{a}}$ recomendación & 530 & 100,0 & ---- & ---- & ---- & ---- \\
\hline
\end{tabular}

Fuente: Elaboración propia 
Tabla 4. Cumplimiento general del Código PAOS por los anuncios de alimentos y bebidas dirigidos a menores de doce años

\begin{tabular}{|c|c|c|c|c|c|c|}
\hline & \multicolumn{2}{|c|}{ Cumplimiento } & \multicolumn{2}{|c|}{ No Cumplimiento } & \multicolumn{2}{|c|}{ Cumplimiento dudoso } \\
\hline & $\mathrm{N}$ & $\%$ & $\mathrm{~N}$ & $\%$ & $\mathrm{~N}$ & $\%$ \\
\hline \multicolumn{7}{|l|}{ Cadena de televisión } \\
\hline Cadena infantil & 65 & 76,5 & 392 & 91,2 & --- & --- \\
\hline Boing & 28 & 33,0 & 211 & 49,1 & ---- & ---- \\
\hline Disney Channel & 37 & 43,5 & 181 & 42,1 & --- & --- \\
\hline Cadenas generalistas & 20 & 23,5 & 38 & 8,8 & 15 & 100,0 \\
\hline Antena3 & 13 & 15,3 & 18 & 4,2 & 11 & 73,3 \\
\hline Telecinco & 7 & 8,2 & 20 & 4,6 & 4 & 26,7 \\
\hline \multicolumn{7}{|l|}{ Día de la semana } \\
\hline Diario & 67 & 78,8 & 330 & 76,7 & 13 & 86,7 \\
\hline Lunes & 12 & 14,1 & 61 & 14,2 & 2 & 13,3 \\
\hline Martes & 11 & 12,9 & 56 & 13,0 & 3 & 20,0 \\
\hline Miércoles & 11 & 12,9 & 62 & 14,4 & 2 & 13,3 \\
\hline Jueves & 13 & 15,3 & 70 & 16,3 & 4 & 26,7 \\
\hline Viernes & 20 & 23,5 & 81 & 18,8 & 2 & 13,3 \\
\hline Fin de semana & 18 & 21,2 & 100 & 23,3 & 2 & 13,3 \\
\hline Sábado & 9 & 10,6 & 46 & 10,7 & 1 & 6,7 \\
\hline Domingo & 9 & 10,6 & 54 & 12,6 & 1 & 6,7 \\
\hline \multicolumn{7}{|l|}{ Tipo de alimento } \\
\hline Comida & 33 & 38,8 & 231 & 53,7 & 14 & 93,3 \\
\hline Bebida & 52 & 61,2 & 199 & 46,3 & 1 & 6,7 \\
\hline \multicolumn{7}{|l|}{ Contenido nutricional } \\
\hline Esencial & 83 & 97,6 & 61 & 14,2 & --- & ---- \\
\hline No esencial & 2 & 2,4 & 369 & 85,8 & 15 & 100,0 \\
\hline Misceláneo & --- & --- & ---- & ---- & --- & --- \\
\hline Total & 85 & 16,0 & 430 & 81,1 & 15 & 2,9 \\
\hline
\end{tabular}

Fuente: Elaboración propia

\section{Conclusiones}

Con el objetivo de evaluar la eficacia del Código PAOS a través del análisis del cumplimiento de sus normas y recomendaciones, se han analizado 530 anuncios de alimentos y bebidas dirigidos al público infantil y emitidos en cuatro cadenas que emiten en abierto, dos dirigidas exclusivamente al público infantil y dos generalistas. Del análisis realizado se ha podido concluir lo siguiente:

1. La elevada exposición de los niños menores de doce años a la publicidad de alimentos y bebidas emitida en los canales infantiles, en comparación con los comerciales expuestos en canales generalistas durante el periodo analizado: 457 anuncios 
frente a 73 emitidos en las cadenas generalistas. Estas diferencias superan las identificadas en 2012 por León-Flández et al. (2017), en la que algo más de la mitad de los anuncios analizados habían sido emitidos en cadenas infantiles. No se observan, sin embargo, diferencias muy notables en la ratio de anuncios de alimentos y bebidas no saludables respecto a los saludables entre cadenas generalistas y cadenas temáticas orientadas específicamente al público infantil. Además, los menores de doce años están más expuestos a estos mensajes los días de diario, con una media de 82 anuncios, y una mayor concentración de estos en el horario de 8:00 a 9:00 horas.

2. Los productos alimenticios emitidos en el horario de protección infantil no son los adecuados para una dieta saludable debido a su escaso valor nutricional, observándose un elevado porcentaje de alimentos calificados como no esenciales y no saludables (72,8\%). Estos resultados confirman la divergencia detectada por Jesús Bermejo (2006) entre el tipo de alimentos que los expertos aconsejan y lo que propone la publicidad. Más preocupante resulta la tendencia creciente en la presencia de publicidad insana en la franja de protección reforzada, con un incremento de 8,7 puntos porcentuales con respecto a 2012, cuando alcanzó el 64.1 \% (León-Flández et al., 2017).

Por otra parte, este análisis ha permitido establecer una relación directa entre el contenido nutricional de los productos anunciados y el cumplimiento del Código: de 430 anuncios que lo han incumplido, la gran mayoría (369) se han clasificado como productos hipercalóricos y excesivos en azúcares, grasas y sal. Esto, unido a los resultados de otros estudios realizados en el ámbito nacional (González, 2013; Royo-Bordonada et al., 2016) e internacional (Huizinga y Kruse, 2016, Jensen y Ronit, 2015), sugiere que son los productos no saludables los que intencionalmente utilizan estrategias más agresivas para fomentar su consumo.

Una novedad que aparece en el tratamiento de los mensajes comerciales en este estudio es que la mayoría de los anunciantes han dado visibilidad a mensajes que fomentan la importancia de adquirir hábitos de vida saludables a través de su adhesión voluntaria al Plan Havisa, promovido por la Agencia Española de Seguridad Alimentaria y Nutrición (AESAN). En este sentido, podemos advertir cómo la publicidad puede ser una gran aliada para contribuir a la información y sensibilización de la población en el desarrollo de ciertas prácticas y estilos de vida deseables.

3. Los anuncios de alimentos y bebidas apoyan su argumento de venta más en el incentivo que en el alimento. La norma 6 es la que presenta el porcentaje más alto de incumplimiento. La mayoría de los anuncios analizados $(81,1 \%)$ han hecho uso de fuertes estrategias persuasivas para incitar a los menores de edad a la compra, a través de la oferta de regalos infantiles y asociando el consumo del producto con la diversión, éxito y prestigio. La vulneración de esta norma ha llegado casi a duplicarse respecto a 2012, cuando 45,1\% de los anuncios hicieron uso de estas técnicas de presión comercial (León-Flández et al., 2017). Por el contrario, se observa un descenso de los incumplimientos de la norma 7 , relativa a la promoción a través de personajes conocidos, respecto al mismo año (León-Flández et al., 2017); siendo también la que registra el porcentaje más bajo de incumplimiento: 15 anuncios frente a 105 que cumplieron con la norma.

4. La efectividad de la autorregulación publicitaria del sector de bebidas y alimentos ha mejorado, a tenor de los datos recopilados y en comparación con los resultados para 2012 del estudio de León-Flández et al. (2017). Aunque el número de anuncios con algún incumplimiento ha sido significativamente más alto que el de aquellos que 
cumplen con todas las normas y recomendaciones, podemos valorar que la calidad publicitaria ha mejorado con respecto al año 2012. En la investigación realizada por León-Flández et al. (2017), solo se encontró una norma que se cumplió plenamente, la norma 11, referida a la seguridad de los productos. En nuestro análisis además de esta norma, todos los anuncios utilizaron un lenguaje acorde al público infantil (norma 5) y respetaron el principio de autenticidad publicitaria (norma 8); asimismo, casi todos los anuncios (93.4\%) han incluido mensajes relativos a hábitos o estilos de vida saludables. Si atendemos a un análisis individual de las normas éticas, se observa un elevado nivel de cumplimiento, que supera a las vulneraciones en cinco de las siete normas analizadas.

De acuerdo con estos resultados, podemos concluir que, aunque se haya observado una mejora en el seguimiento del código PAOS en los seis años que se sitúan entre 2018 y 2012, se hace patente que la autorregulación no constituye un mecanismo efectivo para limitar la exposición de los menores a la publicidad de alimentos y bebidas no básicos y poco saludables. Son, precisamente, los comerciales de este tipo de alimentos los que hacen un uso más intenso de estrategias orientadas a persuadir a los menores de su consumo, incumpliendo el código que voluntariamente firmaron las empresas que las representan. Estos resultados ponen en duda la eficacia de una iniciativa de carácter voluntario de la industria, tal y como denuncian diversos organismos.

Estos resultados son relevantes en un contexto en el que el Ministerio de Sanidad en España ha anunciado, a finales de 2018, diferentes medidas orientadas a la modificación del código PAOS, proponiendo la limitación de la publicidad de productos con perfiles nutricionales bajos. De acuerdo con esto, parece que el ejecutivo español no contempla sustituir el actual sistema de autorregulación por medidas legislativas, como ya se hizo hace en 2010 con el Código de Autorregulación sobre Contenidos Televisivos e Infancia y como diversos colectivos reclaman. Cabe preguntarse si cabe confiar en que un mecanismo, que es ampliamente cuestionado por su eficacia debido a la falta de compromiso real de sus firmantes, obtendrá mejores resultados por el hecho de establecer medidas más restrictivas que afectan, precisamente, al colectivo que más infracciones comete. Por otra parte, también queda por ver cómo se materializan los criterios recogidos en el «EU Pledge» (Compromiso de la UE) para establecer el perfil nutricional de los alimentos y su exclusión de la publicidad televisiva en horario infantil.

Como han demostrado Tarabashkina, Quester y Crouch (2016), los niños se sienten más atraídos por los alimentos menos saludables, aun cuando conocen su contenido nutricional. Además, la investigación empírica demuestra que esta preferencia y los comportamientos de consumo asociados se amplían al usarse determinadas técnicas de comercialización, en particular la publicidad en televisión (Smith, Kelly \& Boyland, 2019). Todo ello parece indicar que la educación en el contenido nutricional de los alimentos, que vendría facilitada por la aparición de numerosas aplicaciones que nos proporcionan esta información, la inclusión de estos contenidos en el sistema educativo y la normativa que obliga a incluir datos al respecto en el envase no son suficientes para garantizar que los niños consuman productos más saludables. Se hace necesario, por tanto, establecer una regulación estricta de la publicidad de alimentos dirigidas a niños, y no confiar en la autorregulación del sector, así como impulsar una correcta alfabetización audiovisual entre los niños para que estos sean capaces de identificar los objetivos persuasivos de la publicidad. 


\section{Bibliografía}

Agencia Española de Consumo, Seguridad Alimentaria y Nutrición (AECOSAN) (2013). Código de correlación de la publicidad de alimentos y bebidas dirigidas a menores, prevención de la obesidad y saludo (Código PAOS). Autocontrol. Recuperado de http://www.aecosan. msssi.gob.es/AECOSAN/docs/documentos/nutricion/Nuevo_Codigo_PAOS_2012_espanol. pdf.

Aranceta, J., Pérez, C., Alberdi, G., Ramos, N. \& Lázaro, S. (2016). Prevalencia de obesidad general y obesidad abdominal en la población adulta española (25-64 años) 2014-2015: estudio ENPE. Revista Española de Cardiología, 69(6):579-587. DOI: 10.1016/j.recesp.2016.02.010

Autocontrol (2018). Trabajamos por una publicidad responsable, 2018. Recuperado de https:// www.autocontrol.es/wp-content/uploads/2016/02/folleto-corporativo-autocontrol.pdf.

Barlovento Comunicación (2018): Análisis televisivo 2018. Recuperado de https://www.barloventocomunicacion.es/wp-content/uploads/2018/12/analisis-televisivo-2018-BarloventoComunicacion.pdf

--- (2018). Análisis mensual del comportamiento de la audiencia televisiva. Recuperado de https://www.barloventocomunicacion.es/audiencias-mensuales/analisis-marzo-2018/.

Bermejo, J. (2006). Mi hijo y la televisión. Madrid, España: Pirámide.

Cairns, G., Angus, K., Hasting, G \& Caraher, M. (2012). Systematic reviews of the evidence on the nature, extent and effects of food marketing to children. A retrospective summary. Appetite, 62, 209-215. doi: 10.1016/j.appet.2012.04.017

Campos, D., Hernández-Torres, J.J., Agil, A., Comino, M., López, J.C., Macías, V. \& Campoy, C. (2016). Analysis of food advertising to children on Spanish television: probing exposure to television marketing. Archives of Medical Science, 12(4), 799-807. doi: 10.5114/ aoms.2016.60969

Caroli, M., Argentieri, L, Cardone, M. \& Masi, A. (2004). Role of television in childhood obesity prevention. International Journal of Obesity, 28, 104-108. doi: 10.1038/sj.ijo.0802802

Dennison, B.A. y Edmunds, L.S. (2008). The role of television in childhood obesity. Progress in Pediatric Cardiology, 25 (2), 191-197. doi: 10.1016/j.ppedcard.2008.05.010

Estudio Aladino (2015). Estudio de Vigilancia del Crecimiento, Alimentación, Actividad Física, Desarrollo Infantil y Obesidad en España 2015. Agencia Española de Consumo, Seguridad Alimentaria y Nutrición. Ministerio de Sanidad, Servicios Sociales e Igualdad. Madrid. Recuperado de http://www.aecosan.msssi.gob.es/AECOSAN/web/nutricion/detalle/ aladino_2015.htm.

Garmendia, M., Jiménez, E., Casado, M.A. \& Macheroni, G. (2016). Net Children Go Mobile: Riesgos y oportunidades en internet y el uso de dispositivos móviles entre menores españoles (2010-2015). Madrid: Red.es/Universidad del País Vasco. https://bit.ly/2PWgtaD.

González, C. (2013). La publicidad dirigida a niños en el sector de la alimentación: un estudio atendiendo al tipo de producto. Historia y Comunicación social, 18, 175-187. Recuperado de http://dx.doi.org/10.5209/rev_HICS.2013.v18.44235

Guthold, R., Cowan, M.J., Autenrieth, C.S., Kann, L. \& Riley, L.M. (2010). Physical activity and sedentary behavior among schoolchildren: a 34-country comparison. Journal of Pediatrics, 157, 1, 43-49. doi: 10.1016/j.jpeds.2010.01.019

Huizinga, O. \& Kruse, M. (2016). Food industry self-regulation scheme «EU Pledge» cannot prevent the marketing of unhealthy foods to children. Obesity Medicine, 1, 24-28. doi: 10.1016/j.obmed.2016.01.004

Icmedia (2014). La protección del menor tras la ley general de la comunicación audiovisual. Perspectivas y retos. Federación de Asociaciones de Consumidores y Usuarios de los Medios. Recuperado de https://www.observatoriodelainfancia.es/oia/esp/documentos_ ficha. aspx?id=3556.

Infoadex (2018). Estudio de la inversión publicitaria en España 2018. Recuperado de http:// www.infoadex.es/home/wp-content/uploads/2018/02/Estudio-InfoAdex-2018.pdf. 
Jensen, JD. \& Ronit, K. (2015). The EU pledge for responsible marketing of food and beverages to children: implementation in food companies. European Journal of Clinical Nutrition, 69 (8), 896-901. doi:10.1038/ejen.2015.52

Kelly, B., Halford, J.C. G., Boyland, E.J, Chapman, K, Bautista-Castaño, I., Berg, C., ... \& Summerbell, C. (2010). Television Food Advertising to Children: A Global Perspective. American Public Health Association, 100 (9), 1730-1736. doi: 10.2105/AJPH.2009.179267

León-Flández, K., Rico-Gómez, A, Moya-Geromin, M. A., Romero-Fernández, M., Bosqued-Estefania, M. J., Damián, J., ... \& Royo-Bordonada, M. A. (2017). Evaluation of compliance with the Spanish Code of self-regulation of food and drinks advertising directed at children under the age of 12 years in Spain, 2012. Public Health, 150, 121-129. doi: 10.1016/j. puhe.2017.05.013

Ley $17 / 2011$, de 5 de julio, de seguridad alimentaria y nutrición. Boletín Oficial del Estado, núm.160, de 6 de julio de 2011. Recuperado de https://www.boe.es/diario_boe/txt.php?id=BOE-A-2011-11604.

Ley 34/1988, de 11 de noviembre, General de Publicidad. Boletín Oficial del Estado, núm.274, de 15 de noviembre de 1988. Recuperado de https://www.boe.es/buscar/act.php?id=BOE-A-1988- 26156\&p=20091231\&tn=1.

Ley $7 /$ 2010, de 31 de marzo, General de la Comunicación Audiovisual. Boletín Oficial del Estado, núm.79, de 1 de abril de 2010. Recuperado de https://www.boe.es/buscar/act. php?id=BOE-A-2010-5292.

Livingstone, S. y Helsper, E. (2006). Does Advertising Literacy Mediate the Effects of Advertising on Children? A Critical Examination of Two Linked Research Literatures in Relation to Obesity and Food Choice. Journal of Communication, 56, 560-584 doi: 10.1111/j.14602466.2006. 00301.x

Martín, M. y Fernández, M. D. (2011). Cinco años de Código PAOS en España: un análisis DAFO. Revista de Comunicación y Salud, 1 (1), 31-41. Recuperado de http://revistadecomunicacionysalud.org/index.php/rcys/article/view/4/58.

McGinnis, J.; Goolman J. y Kraak V. (2006). Food marketing to children and youth: threat or opportunity? Washington, DC: The National Academies Press. Recuperado de https:// zodml.org/sites/default/files/Food_Marketing_to_Children_and_Youth_Threat_or_Opportunity.pdf

Menéndez, R. A. y Franco, F. J. (2009). Publicidad y alimentación: influencia de los anuncios gráficos en las pautas alimentarias de infancia y adolescencia. Nutrición Hospitalaria, 24 (3), 318-325. Recuperado de http://www.redalyc.org/articulo.oa?id=309226746009.

Ofcom (2006a). Television Advertising of Food and Drink Products to Children: Options for New Restrictions. London: Office of Communications. https://www.ofcom.org.uk/_data/assets/ pdf_file/0015/43008/Television-Advertising-of-Food-and-Drink-Products-to-Children.pdf

Organización Mundial de la Salud (2017). La obesidad entre los niños y los adolescentes se ha multiplicado por 10 en los cuatro últimos decenios. Centro de prensa, 11 de octubre. Recuperado de http://www.who.int/mediacentre/news/releases/2017/increase-childhood-obesity/es/.

Pardo, M.M., Lázaro, E.J., García, F.L (2012). Adecuación constitucional y legalidad del discurso publicitario. En E.J. Lázaro (coord.). Derecho de la Publicidad (pp. 91-131). Madrid, España: Civitas.

Ponce-Blandón, J.A.; Pabón-Carrasco, M. y Lomas-Campos, M.M. (2017). Análisis de contenido de la publicidad de productos alimenticios dirigidos a la población infantil. Gaceta Sanitaria, 31 (3), 180-186. DOI: 10.1016/j.gaceta.2016.12.008

Reilly, JJ., Dorosty, A., Ness, A. \& Sherriff, A. (2005). Early life risk factors for obesity in childhood: cohort study. Leading General Medical Journal, 330 (7504), 1357. doi:10.1136/ bmj.38470. 670903.E0

Romero, MM. (2016). Evaluación de la publicidad alimentaria dirigida a niños en televisión en España (tesis doctoral). Universidad Autónoma de Madrid, Madrid, España. Recuperado de 
https://repositorio.uam.es/bitstream/handle/10486/678458/romero_fernandez_mar.pdf?sequence $=1$

Royo-Bordonada, MA., Bosqued-Estefanía, MJ., Damián, J., López-Jurado, L. \& Moya-Geromini, MA. (2016). Nutrition and health claims in products directed at children via television in Spain in 2012. Gaceta Sanitaria, 30, 3, 221-226. doi: 10.1016/j.gaceta.2016.01.004

Royo-Bordonada, M.A. y Rodríguez, F. (2015). Publicidad alimentaria y salud. Estado de la situación en España. Mediterráneo Económico, 27, 319-330. Recuperado de http://www.publicacionescajamar.es/pdf/publicaciones-periodicas/mediterraneo-economico/27/27-738.pdf.

Smith, R., Kelly, B. \& Boyland, E. (2019). Food Marketing Influences Children's Attitudes, Preferences and Consumption: A Systematic Critical Review. Nutrients, 11, 875. doi:10.3390/ nu11040875

Tarabashkina, L., Quester, P. \& Crouch, R. (2016). Food advertising, children's food choices and obesity: interplay of cognitive defences and product evaluation: an experimental study. International Journal of Obesity, 40, 581-586. DOI: 10.1038/ijo.2015.234

Viner, R.M. y Cole, T.J. (2005). Television viewing in early childhood predicts adult body mass index. Journal of Pediatrics, 147 (4), 429-435. DOI: 10.1016/j.jpeds.2005.05.005

Whalen, R., Harrold, J., Child, S., Halford, J. \& Boyland, E. (2018). The Health Halo Trend in UK Television Food Advertising Viewed by Children: The Rise of Implicit and Explicit Health Messaging in the Promotion of Unhealthy Foods. International Journal of Environmental Research and Public Health, 15 (3), 560-568. doi: 10.3390/ijerph15030560

Wiecha, J.L., Peterson, K.E, Ludwig, D.S., Kim, J., Sobo, A. \& Gortmaker, SL. (2006). When Children Eat What They Watch. Impact of Television Viewing on Dietary Intake in Youth. Archives of Pediatrics \& Adolescent Medicine, 160 (4), 436-442. doi: 10.1001/archpedi.160.4.436

Young, B. (1990). Television advertising and children. Oxford, Reino Unido: Oxford University Press. 